\title{
Breast Sparganosis Mimicking Ipsilateral Breast Tumor Recurrence in a Patient with a History of Breast Conserving Surgery: A Case Report
}

\author{
Min Kyung Jeong, ${ }^{1}$ Jun Hyun Baik, ${ }^{1,}{ }^{*}$ Yeong Yi An, ${ }^{1}$ and Hyun Joo Choi ${ }^{2}$ \\ ${ }^{1}$ Department of Radiology, St. Vincent's Hospital, College of Medicine, The Catholic University of Korea, Republic of Korea \\ ${ }^{2}$ Department of Hospital Pathology, St. Vincent's Hospital, College of Medicine, The Catholic University of Korea, Republic of Korea \\ "Corresponding author: Jun Hyun Baik, Department of Radiology, St. Vincent's Hospital, College of Medicine, The Catholic University of Korea, Republic of Korea. Tel: \\ +82-312497484, Fax: +82-312475713, E-mail: jhbaik@catholic.ac.kr
}

Received 2016 August 19; Revised 2016 September 13; Accepted 2016 September 26.

\begin{abstract}
Sparganosis is a rare parasitic infection caused by ingesting plerocercoid larvae in impure water or consuming raw intermediate hosts such as frogs and snakes. The breast is a rare site of infection. Breast sparganosis usually presents as a migrating subcutaneous mass that clinically and radiologically mimics malignancy. Herein, we report a surgically confirmed case of breast sparganosis mimicking ipsilateral breast tumor recurrence (IBTR) in a patient with a history of breast conserving surgery (BCS) for breast cancer with multimodal imaging findings.
\end{abstract}

Keywords: Breast sparganosis, Ipsilateral Breast Tumor Recurrence (IBTR), Mammography, Ultrasound, Magnetic Resonance Imaging, 18F-Fluoro-2-D-Glucose Positron Emission Tomography/Computed Tomography

\section{Introduction}

Sparganosis is a rare parasite infection caused by a migrating plerocercoid larva belonging to the genus Spirometra, which is usually found in East Asia (1). It is transmitted by drinking larvae-containing water or consuming raw secondary intermediate hosts such as frogs or snakes (2). This condition most commonly manifests as migrating subcutaneous nodules and can involve various sites, including the abdomen, genitals, extremities, central nervous system, and chest (3). The breast is a rare site of infection (4). Breast sparganosis may mimic malignancy clinically and radiologically and may lead to misdiagnosis (5). Thus, accurate diagnosis and treatment can be challenging, particularly in cases of breast sparganosis with coexisting or treated breast cancer $(6,7)$. To our knowledge, there has been no case report about breast sparganosis mimicking ipsilateral breast tumor recurrence (IBTR) presented with multimodal imaging findings including mammography, ultrasound, magnetric resonance imaging, and 18F-fluoro-2-D-glucose positron emission tomography/computed tomography. Herein, we report an interesting case of breast sparganosis mimicking IBTR in a patient with a history of breast conserving surgery (BCS) for breast cancer with multimodal imaging findings and a brief literature review.

\section{Case Presentation}

The patient was a 66-year-old woman who had a history of BCS and radiation therapy for invasive ductal carcinoma of the right breast. Histopathological examination revealed a $1 \mathrm{~cm}$ sized, grade II invasive ductal carcinoma and all resected axillary lymph nodes were negative for malignancy. Immunohistochemistry staining for estrogen receptor (ER), progesterone receptor (PR), and human epidermal growth factor receptor (HER-2) were all negative. The patient underwent postoperative surveillance for four years and was doing fine, until sternal metastasis and metastatic lymph nodes in the right lower neck, right supraclavicular, mediastinal, and right internal mammary regions were detected on 18F-fluoro-2-Dglucose(18FDG) positron emission tomography/computed tomography (PET/CT). Subsequently, the patient underwent adjuvant chemotherapy with docetaxel and doxorubicin. On follow-up 18FDG PET/CT after six cycles of systemic chemotherapy, 18FDG uptake of all metastatic foci had regressed. Three weeks before her admission, she visited our hospital for post-treatment surveillance. She did not complain of any symptoms and breast examination revealed no breast lumps. However, mammography revealed a newly developed asymmetry with thickening of the overlying skin in the right mid outer breast (Figure 1). Ultrasound showed an indistinct irregular hypoechoic structure surrounded by a poorly-defined hyperechoic lesion in the subcutaneous fat layer of the right lower outer breast and edematous change of the overly- 
ing skin. Dynamic contrast enhanced magnetic resonance imaging (DCE-MRI) revealed a clustered ring enhancement involving the skin and subcutaneous fat tissue in the right lower outer breast. These lesions had slightly high signal intensity on T1-weighted images and low signal intensity on T2-weighted images. The differential diagnosis includes postoperative fat necrosis, abscess, and IBTR. However, postoperative fat necrosis characteristically occurs at the treated site, and it is usually isointense to fat elsewhere in the breast with adjacent enhancement on MRI. Although breast abscesses were present as rim enhancement on MRI, the patient did not complain of any inflammatory symptoms such as sensation of warmth, redness, or painful swelling. Considering her history of breast cancer, IBTR was suspected. 18FDG-PET/CT revealed nodular lesions with mildly increased FDG uptake that had a maximal standardized uptake value (SUVmax) of 2.1 in the right lower outer breast. Surgical excision was performed and the pathologic findings confirmed sparganosis with granulomatous inflammation and multinucleated giant cells.

We retrospectively reviewed previous serial follow-up breast MRIs. The postoperative follow-up MRI two years prior to the excision showed focal clustered ring enhancement with similar features to the current lesion, but was more deeply located within the breast parenchyma (Figure 2). At that time, this lesion was misinterpreted as postoperative fat necrosis developed after breast surgery, and the larva was identified after migrating toward the surface of the breast over a two-year period. Our patient denied eating either raw frogs or snakes; therefore, ingestion of contaminated spring water may be the possible route of sparganosis infection in this patient.

\section{Discussion}

Sparganosis is a rare parasite infection which is usually found in East Asia (1). To the best of our knowledge, 41 cases of breast sparganosis have been reported based on the Korean Medical (KM) database and the Korean association of medical journal editors (KAMJE) database, which were searched to include information from Korean journals that are not included in international citation indexes $(3,4,6,8-12)$. It is transmitted by drinking larvaecontaining water or consuming raw secondary intermediate hosts such as frogs or snakes (2). Spargana can move to any site of the body such as the abdomen, scrotum, chest wall, lower extremities, brain, and rarely involve the breast $(3,4)$. Sparganosis most commonly manifests as a migrating palpable soft tissue nodule without evidence of inflammatory reaction such as warm sensation or painful swelling(4). Thus, it may be confused with malignancy and accurate preoperative diagnosis can be difficult in many cases.

Although breast sparganosis is extremely rare, previously reported cases have described its characteristic radiological findings $(1,3,4,6,8-10)$. The mammographic findings of the breast sparganosis are irregular, lobular or tubular structures or amorphous solid masses (3, $4,6,9,10)$. The ultrasonographic findings of breast sparganosis are elongated, folded, tubular hypoechoic structures and serpiginous echogenic lesions, which correlate with pathologic findings $(1,3,4,6,8-10)$. Hypoechoic tubular structures on ultrasonography correlate with the empty tract left by migration of the larvae; whereas, echogenic lesions correspond to living worms or debris (8). Thus, the diagnosis of breast sparganosis is not difficult if radiologists are familiar with these unique ultrasonographic features. Our case did not represent these typical mammographic and ultrasonographic features of breast sparganosis and mimicked breast malignancy, except for subcutaneous location and thickening of overlying skin that can be helpful for distinguishing breast spargnosis from breast malignancy.

To the best of our knowledge, only two case reports describing DCE-MRI findings of the breast sparganosis have been reported $(11,12)$. In these previous reports, the MRI appearance of breast sparganosis includes multiple round and elongated tubular-shaped lesions showing rim enhancement with persistent kinetic patterns in the subcutaneous fat on post-contrast $\mathrm{T} 1$ weighted images. We also observed similar MRI findings of breast sparganosis in our case. Although the internal characteristic of rim enhancement on DCE-MRI has a 79\% - 92\% positive predictive value for breast cancer (13), benign conditions such as an inflamed cyst, fat necrosis or abscess can also cause rim enhancement. In this case, inflammation of the surrounding subcutaneous fat tissue induced by migrating worms generated various enhancing patterns on DCE-MRI. Only two case reports have described PET/CT findings of breast sparganosis. Ha et al. (11) reported a case of breast sparganosis incidentally detected by PET/CT with a focal FDG uptake and a standardized uptake values (SUV) max of 1.2, which was not as high as malignancy or active inflammation. However, a case of axillary sparganosis reported by Lee et al. (7) showed a hypermetabolic mass with a SUVmax of 7.2. Our case showed mildly increased FDG uptake with a SUVmax of 2.1. Ha et al. (11), Lee et al. (7), and our case showed different degrees of FDG uptake on PET/CT. The degree of 18FDG uptake on PET/CT seems to have a broad spectrum according to parasite type, infection site, host immune status, and inflammatory response.

Although the radiological findings of breast sparganosis have been established, diagnosis can be 

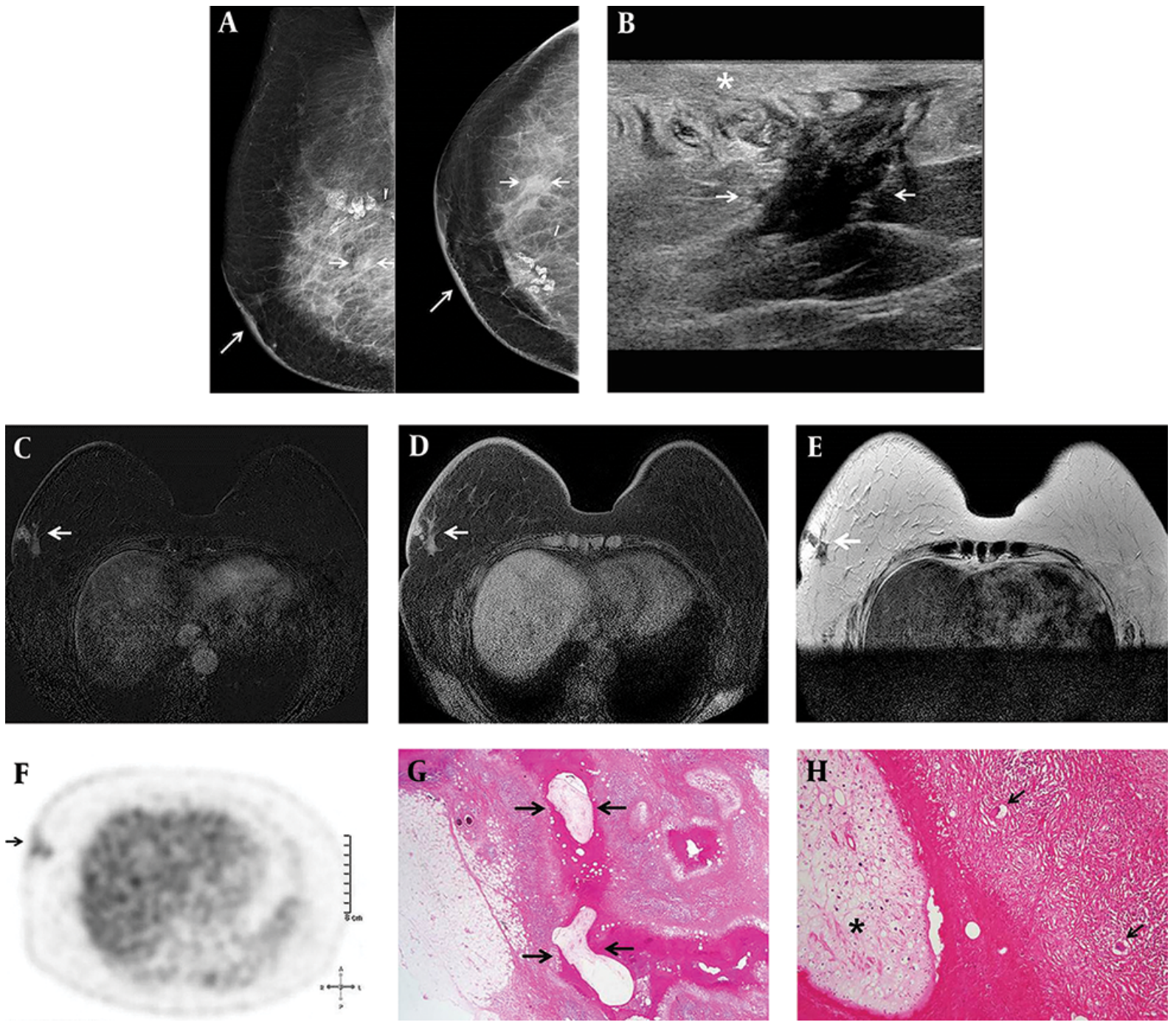

Figure 1. A 66-year-old woman with a history of right upper inner breast cancer was treated with breast conserving surgery. A, Six years after her surgery, annual imaging follow-up showed developing asymmetry (short arrows) at the right mid outer breast with thickening of overlying skin (long arrows) on mammography; B, An indistinct irregular hypoechoic structure (arrows) surrounded by a poorly- defined hyperechoic lesion in the subcutaneous fat layer of the right lower outer breast and edematous change of the overlying skin (star) on ultrasonography; C, Clustered ring enhancement (arrow) involving the skin and subcutaneous fat tissue in the right lower outer breast on post-contrast T1-weighted images; D, Slightly high signal intensity (arrow) on T1-weighted images; E, Low signal intensity (arrow) on T2-weight images of Dynamic contrast enhanced magnetic resonance imaging (DCE-MRI); F, Mildly increased fluoro-D-glucose (FDG) uptake with a SUV max of 2.1 (arrow) in the right lower outer breast on positron emission tomography/computed tomography (PET/CT); G, The pathologic findings confirmed parasite (sparganum) infestation (arrows) in granulomatous inflammation (Hematoxylin-Eosin, $\times 4$ ); H, Sparganum (star) shows eosinophilic folded tegument, subtegumental muscle fibers, and calcospherules, with multinucleated giant cells (arrows) and granulomatous inflammation (Hematoxylin-Eosin, $\times 20$ ).

confusing, particularly in cases of newly diagnosed breast cancer or patients who have undergone treatment for breast cancer $(7,12)$. Lee et al. (12) reported a case of breast sparganosis in a patient with coexisting ipsilateral breast cancer, which mimicked a multicentric malignancy. Recently, Lee et al. (7) also reported a case of multiple axillary lymph node sparganosis that presented as a newly developed axillary mass on follow-up MRI and PET/CT during neoadjuvant chemotherapy. In this case, the main breast cancer lesion exhibited a partial response to chemotherapy, but newly detected axillary lymph node sparganosis on MRI and PET/CT was mistaken for a new, axillary lymph node metastasis of the breast cancer, resulting in a treatment change from chemotherapy to surgery. Correlation with clinical history, the presence of inflammatory symptoms, lesion location, and US and MRI features can be helpful for differential diagnosis. In particular, we think that a subcutaneous location and 


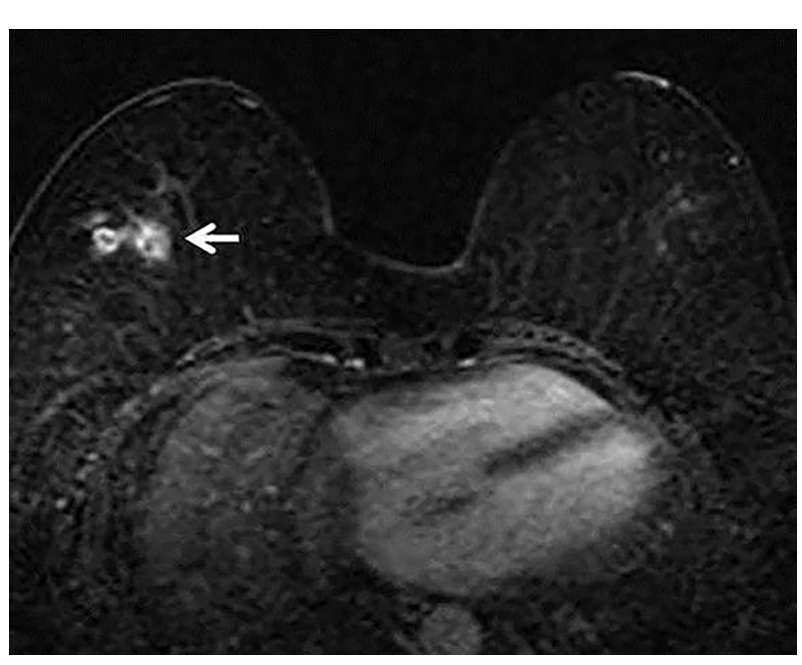

Figure 2. Retrospectively, the postoperative follow-up breast MRI two years prior to the excision showed focal clustered ring enhancement (arrow) with similar features to the current lesion, but was more deeply located within the breast parenchyma.

migration of the lesion on follow-up imaging studies are key distinguishing features of sparganosis in the treated breast.

When sparganosis is suspected, complete surgical removal is the treatment of choice rather than percutaneous biopsy procedures, including core needle biopsy or vacuum-assisted biopsy (4). Percutaneous biopsy procedures may result in worm fragmentation, preventing complete surgical removal and possibly promoting recurrence.

In conclusion, breast sparganosis is a rare parasite infection that may mimic malignancy both clinically and radiologically. Therefore, diagnosis can be difficult, particularly in breast cancer patients. It should be included in the differential diagnosis for incidental breast masses in patients with a history of BCS. A subcutaneous, migrating breast mass with characteristic US and MRI features is distinctive in differentiating breast sparganosis from breast malignancy.

\section{Acknowledgments}

None declared.

\section{Footnotes}

Authors' Contributions: None declared.

Financial Disclosure: None declared.

Funding/Support: None declared.

\section{References}

1. Kim HS, Cha ES, Kim HH, Yoo JY. Spectrum of sonographic findings in superficial breast masses. J Ultrasound Med. 2005;24(5):663-80.

2. Cho JH, Lee KB, Yong TS, Kim BS, Park HB, Ryu KN, et al. Subcutaneous and musculoskeletal sparganosis: imaging characteristics and pathologic correlation. Skeletal Radiol. 2000;29(7):402-8. [PubMed: 10963426].

3. Park JH, Chai JW, Cho N, Paek NS, Guk SM, Shin EH, et al. A surgically confirmed case of breast sparganosis showing characteristic mammography and ultrasonography findings. Korean J Parasitol. 2006;44(2):151-6. doi: 10.3347/kjp.2006.44.2.151. [PubMed: 16809964].

4. Moon HG, Jung EJ, Park ST. Breast sparganosis presenting as a breast mass with vague migrating pain. JAm Coll Surg. 2008;207(2):292. doi: 10.1016/j.jamcollsurg.2008.01.067. [PubMed: 18656060].

5. Kim HY, Kang CH, Kim JH, Lee SH, Park SY, Cho SW. Intramuscular and subcutaneous sparganosis: Sonographic findings. J Clin Ultrasound. 2008;36(9):570-2. doi:10.1002/jcu.20480. [PubMed: 18431747].

6. Hong SJ, Kim YM, Seo M, Kim KS. Breast and scrotal sparganosis: sonographic findings and pathologic correlation. J Ultrasound Med. 2010;29(10):1627-33.

7. Lee EK, Yoo YB. Axillary sparganosis which was misunderstood lymph node metastasis during neoadjuvant chemotheraphy in a breast cancer patient. Ann Surg Treat Res. 2014;87(6):336-9. doi: 10.4174/astr.2014.87.6.336. [PubMed: 25485243].

8. Choi SJ, Park SH, Kim MJ, Jung M, Ko BH. Sparganosis of the breast and lower extremities: sonographic appearance. J Clin Ultrasound. 2014;42(7):436-8. doi:10.1002/jcu.22146. [PubMed: 24633936].

9. Chung SY, Park KS, Lee Y, Park CK. Breast sparganosis: mammographic and ultrasound features. J Clin Ultrasound. 1995;23(7):447-51. [PubMed: 7560161].

10. Koo M, Kim JH, Kim JS, Lee JE, Nam SJ, Yang JH. Cases and literature review of breast sparganosis. World J Surg. 2011;35(3):573-9. doi: 10.1007/s00268-010-0942-1. [PubMed: 21203758].

11. Ha S, Oh SW, Kim YK, Yi A, Ahn YJ. Breast Sparganosis Incidentally Detected by FDG PET/CT. Nucl Med Mol Imaging. 2013;47(2):130-3. doi: 10.1007/s13139-012-0187-z. [PubMed: 24900094].

12. Lee YJ, Choo KS, Bae YT, Kim JY. A Case Report of Breast Sparganosis in a Patient with Ipsilateral Breast Cancer: MRI and Ultrasonographic Findings. J Korean Soc Radiol. 2010;63(6):569-75. doi: 10.3348/jksr.2010.63.6.569.

13. Jeh SK, Kim SH, Kim HS, Kang BJ, Jeong SH, Yim HW, et al. Correlation of the apparent diffusion coefficient value and dynamic magnetic resonance imaging findings with prognostic factors in invasive ductal carcinoma. J Magn Reson Imaging. 2011;33(1):102-9. doi 10.1002/jmri.22400. [PubMed: 21182127]. 